\title{
1 Climate, crypsis and Gloger's rule in a large family of tropical passerine birds
}

\section{2 (Furnariidae)}

3 Rafael S. Marcondes ${ }^{1,2,3}$, Jonathan A. Nations ${ }^{1,3}$, Glenn F. Seeholzer ${ }^{1,4}$ and Robb T. Brumfield ${ }^{1}$

4 1. Louisiana State University Museum of Natural Science and Department of Biological Sciences. Baton Rouge LA, 70803.

2. Corresponding author: rmarco3@1su.edu

3. Joint first authors

4. Current address: Department of Ornithology, American Museum of Natural History, Central Park West at 79th Street, New York, NY, 10024, USA

11 Author contributions: RSM and JAN conceived the study, conducted analyses and wrote the

12 manuscript. RSM and GFS collected data. All authors edited the manuscript. RTB provided

13 institutional and financial resources.

14 Running head: Climate and habitat type in Gloger's rule

15 Data accessibility statement: Color data is deposited on Dryad under DOI

16 10.5061/dryad.s86434s. Climatic data will be deposited on Dryad upon acceptance for

17 publication.

18 Key words: Gloger's rule; Bogert's rule; climate; adaptation; light environments; Furnariidae, 19 coloration; melanin; thermal melanism. 


\section{Acknowledgments}

This work was partially supported by NSF grant DEB-1146265 to

27 RTB and by a "Science Without Borders" doctoral fellowship from Brazil's National Council for

28 Scientific and Technological Development to RSM (CNPq; 201234/2014-9).

29

30

31

32

33

34

35

36

37

38

39

40

41

42

43

44

45

46

47

48 


\section{Abstract}

50 Gloger's rule predicts endothermic animals should have darker colors under warm and

51 rainy climates, but empirical studies have typically found that animals tend to be darker under

52 cool and rainy climates. Moreover, Gloger's rule has rarely been tested jointly with the

53 prediction that animals occupying dark habitats should have darker colors to enhance crypsis.

54 We aimed to disentangle the effects of climate and light environments (habitat type) as correlates

55 of plumage brightness in a large Neotropical passerine family. We found that cooler and rainier

56 climates are associated with darker plumage, even after accounting for habitat types, and that

57 darker habitats are associated with darker plumage, even after accounting climate. There was an

58 important interaction between precipitation and temperature, whereby the negative effect of

59 temperature on brightness becomes stronger under cooler temperatures. Climate and light

60 environments have separate but complementary effects in driving macroevolutionary patterns of

61 plumage color variation in birds.

62

63

64

65

66

67

68

69

70

71 
Ecogeographic rules describe correlations between organismal phenotypes and features

73 of their environment. Their repeated observation across taxa and space is prima facie evidence

74 that they are driven by common selective pressures (Mayr 1963, James 1991, VanderWerf 2011).

75 Gloger's rule (Gloger 1833, Rensch 1929) is a longstanding ecogeographic rule describing a

76 correlation between the colors of mammals and birds and the climatic conditions they occupy.

77 Recent interpretations of Gloger's rule (Delhey 2019, Marcondes et al. in review) have divided it

78 into two versions: one "simple" and the other "complex". The simple version relates to variation

79 in overall melanin content, with greater amounts of melanin making feathers and fur darker

80 (McGraw et al. 2005). This version of Gloger's rule predicts that animals tend to be darker in

81 rainy and warm climates and brighter in dry and cool climates (Gloger 1833). The complex

82 version of Gloger's rule concerns variation specifically in pheomelanin content (Delhey 2019),

83 with greater amounts of pheomelanin making feathers and fur more brown or reddish-brown

84 (McGraw et al. 2005). This paper concerns only the simple version of Gloger's rule, which

85 historically has been the only version most investigators have recognized (Delhey 2019).

86 Gloger's rule has been investigated mostly at the intraspecific level, where evidence is

87 abundant (Zink and Remsen 1986, Delhey 2019). Well-studied examples of species that have

88 been found to be darker in more humid climates come from a broad variety of bird clades and

89 include, but are not limited to, the Barn Owl Tyto alba (Roulin and Randin 2015, Romano et al.

90 2019), Black Sparrowhawk Accipiter melanoleucos (Tate and Amar 2017), Song Sparrow

91 Melospiza melodia (Burtt and Ichida 2004) and Variable Antshrike Thamnophilus caerulescens

92 (Marcondes et al. in review).

93 In contrast, Gloger's (1833) prediction that animals should be darker in warmer climates

94 has rarely been supported (Delhey 2019). More often, it has been found that populations

95 inhabiting warmer climates tend to be lighter than their counterparts from cooler locales, a 
pattern dubbed Bogert's rule and often attributed to thermoregulatory advantages (Clusella-

97 Trullas et al. 2007, Rising et al. 2009, Delhey 2019).

Mayr (1956) argued that ecogeographic rules typically apply only to variation between at the interspecific level. In fact, interspecific comparative analyses are crucial to revealing how evolutionary processes operating within species can be generalized across macroevolutionary scales (Meiri 2011, Stoddard et al. 2019). The predicted negative correlation between brightness and humidity has been supported in phylogenetic comparative studies of the world's primates

104 (Kamilar and Bradley 2011), a large clade of Holartic shrews (Stanchak and Santana 2018), the 105 entire Australian avifauna (Delhey 2018), the world's woodpeckers (Miller et al. 2019), and the world's passerine birds (Delhey et al. 2019). The latter two studies also supported the prediction of Bogert's rule that animals are lighter in warmer regions. of variation in animal color, particularly brightness, is habitat type, or light environment. Endler (1993) predicted that, to enhance crypsis, animals inhabiting dark light environments (e.g. the

111 interior of dense forests) should be darker than those inhabiting open areas with bright light

112 conditions (e.g., savannas), a prediction that has received wide support from comparative studies

113 on birds (McNaught and Owens 2002, Gomez and Thery 2004, Dunn et al. 2015, Maia et al.

114 2016, Shultz and Burns 2017, Marcondes and Brumfield 2019). Because forests, particularly

115 tropical rainforests, are more prevalent in rainier climates, this raises the possibility that the

116 tendency for birds to be darker in more humid places (Gloger's rule) is confounded by a need for

117 crypsis in darker environments. 
120

121

122

123

124

125

126

127

128

129

130

131

132

133

134

135

136

137

138

139

140

141

142

143

driving interspecific variation in plumage brightness. Throughout the Neotropics, furnariids occupy virtually every terrestrial biome and habitat type (here construed to mean the spatial vegetation structure and density typically occupied by each bird species). They are found at the extremes of both precipitation and temperature in the Neotropics, from the warm and rainy

Amazonian rainforests to warm and arid Chaco savannas, and from cool and dry high-elevation puna grasslands to the cool and rainy Andean cloud forests (Remsen 2003). Moreover, even under the same climatic conditions at a single geographic locality, species in this family specialize in such different habitat types as, for example, the lower strata of tropical rainforests, the forest canopy, and patches of open vegetation. They therefore experience dramatically different light environments, from the dim forest understory to intensely sun-lit fields and savannahs. Despite this ecological diversity, furnariids are virtually all festooned exclusively in innumerous shades of brown and rufous that vary relatively little in hue, but greatly in brightness. For example, furnariid colors range from light and creamy brown in the puna- and desert-inhabiting Ochetorhynchus earth-creepers to dark and rich brown in some species of tropical rainforest-dwelling Xiphorhynchus woodcreepers.

If Gloger's rule is driven primarily by climate, species inhabiting rainy and warm climatic regimes are predicted to be darker than those from dry and cool regimes, regardless of their habitat preference. In contrast, if Gloger's rule is mainly a result of birds adapting to be darker in darker (forest) habitats, bird species occupying forest habitats are predicted to be darker than their nonforest-based relatives, even if they inhabit similar climatic regimes. Marcondes and Brumfield (2019) previously demonstrated that furnariid species have evolved to be darker in darker habitats, consistent with Endler's (1993) predictions for crypsis. Here, we sought to investigate the interacting roles of climate and habitat type in driving interspecific variation in plumage brightness in the Furnariidae. 


\section{Methods}

\section{Color data}

We used the color dataset previously described in Marcondes and Brumfield (2019) and

148 deposited on the Dryad digital repository under DOI 10.5061/dryad.s86434s (embargoed until

149 July 16, 2021). Briefly, this dataset includes reflectance data for 250 (84\%) furnariid species,

150 with an average of 6.4 specimens per species (range: 1-8). For each specimen, this dataset

151 includes reflectance spectra from seven plumage patches divided into a dorsal (crown, back,

152 rump and tail) and a ventral (belly, breast and belly) set. We calculated plumage brightness

153 (percentage of reflected white light) and averaged it across all specimens of each species.

154 Because the Furnariidae are sexually monochromatic with no evidence of cryptic sexual

155 dichromatism (Remsen 2003; Tobias et al. 2012; Diniz et al. 2016; Marcondes and Brumfield

156 2019), we considered the sexes together in our analyses. Finally, we used principal component

157 analysis (PCA) to reduce our dataset to one principal component for the venter and one for the

158 dorsum. The first principal components of dorsal and ventral PCAs were both loaded in the same

159 direction by brightness in all plumage patches, thus representing overall brightness of that body

160 surface; subsequent principal components captured various aspects of contrasts between plumage

161 patches within each body surface (Marcondes and Brumfield 2019). Our final color dataset thus

162 consisted of the first principal component score of brightness for each body surface (hereafter,

163 simply "dorsal brightness" and "ventral brightness") for each species.

\section{Habitat and climatic data}

We used Marcondes and Brumfield's (2019) categorization scheme for habitat types,

167 which was based on Endler's (1993) discussion of natural light environments. In brief, each of 
the 250 furnariid species we analyzed was assigned to one habitat type, in decreasing order of ambient light intensity: nonforest, intermediate and forest. The forest category includes only species that occupy the dimly-lit middle and lower strata of rainforests; we assigned canopy and

171 edge species to the intermediate category because these areas are more intensely illuminated than

172 the forest interior (Endler 1993, Marcondes and Brumfield 2019).

173 To obtain climatic data for each furnariid species we used the georeferenced locality

174 records dataset of Seeholzer et al. (2017). This extensively-vetted dataset contains 23,588

175 occurrence records (average $=70.4$ records/species) gathered from museum specimens, audio

176 recordings and observational records. For each locality in this dataset, we obtained mean annual

177 temperature and mean annual precipitation from the BioClim database (Hijmans et al. 2005) and,

178 for each species, we took the median of temperature and precipitation across all its occurrence

179 localities. Because of their different magnitudes and units $\left({ }^{\circ} \mathrm{C}\right.$ for temperature and mm/year for

180 precipitation), before fitting any statistical models (see below), at this stage we scaled each

181 climatic variable to have a mean of 0 and a standard deviation of 1.

\section{Statistical analyses}

To test the two hypotheses regarding the effects of climate and habitat on plumage

185 brightness in the Furnariidae, we fit a series of phylogenetic Bayesian multilevel linear models

186 using the modeling software Stan (Carpenter et al. 2017) as implemented in the R library brms

187 (Bürkner 2017). All R scripts used for the analyses are available at https://github.com/jonnations.

188 The multilevel model framework allowed us to fit linear models with multiple predictor variables

189 while including group-level effects that account for statistical non-independence of species data

190 due to shared phylogenetic history. 
First, we tested the hypothesis that Gloger's rule is primarily driven by climate, and species in wetter and warmer localities are darker than those from drier and cooler localities, 193 regardless of habitat preference (forest, intermediate or nonforest). Under this hypothesis, we expect that species occupying wet and warm locales will be darker than those from dry and cool locales even when comparing nonforest species in the former to forest species in the latter. We fit two identical phylogenetic multivariate linear regression models, one with dorsal brightness as our response variable (Dorsal Model 1) and the other with ventral brightness as our response variable (Ventral Model 1). These models use precipitation, temperature, and the interaction between precipitation and temperature as the predictor variables. We used a species level matrix of scaled phylogenetic branch lengths (i.e., the phylogenetic correlation matrix; Bürkner 2017)

201 from the phylogeny of Harvey et al. (in review) as a group-level effect (de Villemereuil et al.

202 2012) to account for correlations due to phylogenetic relatedness of species. These models test

203 the Gloger's rule prediction that birds occupying warm and rainy regions should be darker than

204 those occupying cool and dry regions (Gloger 1833, Rensch 1929). A nonzero, negative effect of

205 precipitation on brightness would be consistent with Gloger's rule, and a nonzero, positive effect

206 would contradict it; likewise for the effect of temperature on brightness. Dorsal and Ventral

207 Models 1 also estimate the interaction parameter of temperature and precipitation, which allows

208 us to explicitly test whether wetter and warmer habitats result in darker plumage, and drier,

209 cooler habitats result in brighter plumage. As both the dorsal and ventral brightness data had a

210 slight positive skew, we used the skew-normal distribution family to describe the response

211 variable rather than a simple Gaussian distribution. This distribution family estimates an

212 additional parameter, alpha, which describes the direction and the strength of the skew. We fit

213 regularizing priors on the group-level effects to prevent MCMC chains from occasionally

214 searching very large, unreasonable values of model space (Gelman 2006; McElreath 2016). Each 
215 of the models included 4 chains run for 10000 generations with 5000 generations of warm-up

216 and 5000 chains of sampling. We assessed chain convergence using the Gelman-Rubin

217 diagnostic $\hat{R}$, and chain efficiency using effective sample size (ESS). $\hat{R}<1.01$ and ESS $>500$

218 represent acceptable convergence and mixing.

219 We also tested the alternative hypothesis that Gloger's rule is related to light

220 environments regardless of climatic variables. Under this hypothesis, we expect species

221 occupying forested habitats to be darker than those inhabiting nonforest habitats, even if the

222 nonforest species are in rainier and warmer climates. Specifically, we separated the effects of

223 habitat from the effects of climate by fitting a phylogenetic multiple regression linear model with

224 dorsal brightness (Dorsal Model 2) or ventral brightness (Ventral Model 2) as our response and

225 temperature, precipitation, and our categorical habitat as predictor variables. As in Dorsal and

226 Ventral Models 1, we included our phylogenetic correlation matrix as a group level effect.

227 Dorsal and Ventral Models 2 have three predictor variables, generating three population-level

228 outcomes: 1) The effect of precipitation on brightness, corrected for the influence of temperature,

229 habitat, and phylogeny, 2) the effect of temperature on brightness, corrected for precipitation,

230 habitat, and phylogeny, and 3) a posterior distribution of the mean brightness values of each

231 habitat conditioned on the phylogenetic relationships, and corrected for the effects of

232 precipitation and temperature. The estimated mean brightness values can directly address our

233 question about whether species in darker habitats have darker plumage regardless of their

234 climatic regimes. For categorical predictors, brms assigns a random category (habitat type in our

235 case) as a dummy variable to use as the intercept value, so we removed the intercept parameter

236 from the model to directly generate posterior distributions for each habitat. We used these

237 posterior distributions of the mean brightness for each habitat to determine if species in different

238 habitats differ in their brightness. To determine if the posterior distributions of the mean 
239 brightness for each habitat are different from one another, we calculated the distributions of the

240 differences of each habitat's brightness estimates, i.e. contrasts (nonforest-intermediate,

241 nonforest-forest, intermediate-forest) (McElreath 2016; Roycroft et al. 2019) using the

242 compare_levels function in the R library tidybayes (Kay 2019). If the 95\% credible interval of

243 these difference distributions does not overlap zero, then we can credibly say that brightness is

244 different between those habitats. This method of calculating the differences between posterior

245 distributions is analogous to the "Bayesian T-Test" of Kruschke (2013). As in Model 1, we used

246 regularizing priors and ran 4 chains of 10,000 generations and checked for convergence with $\hat{R}$

247 and ESS.

248 As a null model, we also fit an intercept-only phylogenetic multilevel model for

249 brightness. This model has no predictor variables and only estimates the intercept of the group

250 level-effect, in our case the phylogenetic correlation matrix. For each plumage surface (dorsal

251 and ventral), we performed model comparison of our three models using the difference in

252 expected log predictive density (ELPD) from the widely applicable information criteria (WAIC,

253 Wantanabe 2010) using the waic function in the R package loo (Vehtari et al 2018), which

254 calculates the ELPD and the standard error of the estimate. WAIC is appropriate for Bayesian

255 inference with non-Gaussian posterior distributions (Gelman, Hwang, and Vehtari 2013); lower

256 WAIC values represent greater support for a model. Comparing against the null allowed us to

257 verify that precipitation, temperature, and habitat improved the predictive ability of our model

258 rather than phylogeny alone explaining differences in brightness. WAIC also allowed us to

259 assess whether the climate interaction model or the climate + habitat model was a better

260 predictor of our brightness data.

\section{Results}


All of our Bayesian multilevel models properly converged, and all parameters had $\widehat{R}<$

2641.01 and ESS $>500$. Results of Model 1 for the dorsal plumage showed a strong negative effect

265 of precipitation on dorsal plumage brightness (Table 1, Figure 1), indicating that as precipitation

266 increases, plumage gets darker, as predicted by Gloger's rule. Model 1 also showed a strong

267 positive effect of temperature on dorsal plumage (Table 1, Figure 1), demonstrating that as

268 temperature increases, dorsal plumage gets brighter, contra Gloger's rule but consistent with

269 Bogert's rule. We also found a positive interaction between precipitation and temperature (Table

270 1, Figures 1 and 2). This interaction indicates that the negative effect of precipitation on

271 brightness decreases with increasing temperature. In other words, Gloger's rule is more notable

272 when comparing species that vary in the amount of precipitation they receive but all occupy

273 similarly cool environments, rather than when comparing species that vary in precipitation but

274 which all occupy similarly warm environments.

275 We found similar results for the ventral plumage. Precipitation had a negative effect on

276 ventral plumage brightness (Table 1, Figure 1). Temperature had an uncertain positive effect on

277 ventral brightness (95\% credible interval overlapping 0), demonstrating that temperature has less

278 importance on ventral plumage than on dorsal plumage. We found a weak positive effect of the

279 interaction between precipitation and temperature on the ventral plumage (Table 1, Figures 1 and

280 2).

281 There was a subtle difference between the venter and the dorsum in effects of the

282 interaction of precipitation and temperature and brightness (Figure 2). Both plumage surfaces

283 tended to be darkest for species in cool/rainy climates and brightest in cool/dry climates. But

284 whereas temperature seemed to have little effect on dorsal brightness in dry climates, ventral

285 plumages tended to be darker under warm/dry conditions than under cool/dry conditions. In other

286 words, there is little change in dorsal brightness when comparing species from cool/dry and 
287 warm/dry conditions, but ventral brightness is higher (lighter) in species from cool/dry than 288 warm/dry conditions.

289 Our Dorsal and Ventral Models 2, in which we removed the interaction between

290 precipitation and temperature and added habitat as a predictor, showed a similar negative effect

291 of precipitation on both dorsal plumage and ventral plumage (Table 2). We also found positive

292 effects of temperature on dorsal, and an uncertain effect of temperature on ventral plumage

293 (Table 2). This model also estimated the posterior distributions of mean plumage brightness for

294 each habitat, conditioned on phylogenetic effects and the climatic variables (Figure 3). We then

295 calculated the differences of those distributions (Figure 3). For the dorsal plumage we found that

296 intermediate and nonforest species are credibly brighter than forest species but not

297 distinguishable from each other, because the difference in their posterior distributions overlapped

298 zero (Figure 3). Results were similar for ventral plumage, except that the difference between

299 intermediate and forest species slightly overlapped zero. Therefore, we found that birds in forest

300 habitats are darker than birds from intermediate or nonforest habitats, even after accounting for

301 differences in temperature and precipitation.

We used the ELPD scores of the WAIC analysis to compare Models 1 and 2 to the null.

303 We found that both predicted dorsal plumage brightness better than the null model (Table 3).

304 However, the standard error of the ELPD scores of Models 1 and 2 overlapped, so that we are 305 unable to conclude which of these two models better predicted dorsal plumage brightness. For

306 the ventral plumage, Models 1 and 2 also better predicted ventral brightness than the null model,

307 but in this case Model 2, which included habitat, was a better predictor of ventral plumage

308 brightness than Model 1, which did not include habitat. 
Gloger's rule is a longstanding ecogeographic principle predicting that birds and

312 mammals that inhabit rainier and warmer climates tend to have darker plumage and pelage color

313 than their counterparts (intra- as well as interspecific) from drier and cooler places (Gloger 1833,

314 Rensch 1929, Mayr 1942, 1963, Delhey 2017, 2019). Here, we found strong support in the

315 Furnariidae for the predicted relationship between brightness and precipitation. In contrast, we

316 found that furnariid species tended to be darker in cooler climates, contrary to the second

317 prediction of Gloger's rule, but consistent with a pattern dubbed Bogert's rule or thermal

318 melanism, which is often observed in ectothermic animals (Clusella-Trullas et al. 2018). We also

319 found a credible positive interaction between precipitation and brightness, meaning that the

320 negative relationship between precipitation and plumage brightness becomes stronger in cooler

321 climates (Figures 1 and 2). Finally, forest-based lineages tended to have darker plumage than

322 nonforest-based lineages (Figure 3), consistent with a previous study on furnariids and other

323 closely-related families (Marcondes and Brumfield 2019). But, here, we expanded on that

324 previous finding by showing that that tendency for birds to have darker plumage in darker

325 habitats persists even after accounting for the effects of climate. This indicates that climate and

326 light environments have separate but complementary effects in driving macroevolutionary

327 patterns of plumage color variation in birds.

328

\section{Gloger's rule, precipitation and temperature}

Gloger (1833) wrote that "melanins [...] increase with higher temperature and humidity"

331 (translation from the German from Delhey 2019), implicating both climatic variables in the rule

332 that would become his namesake. But Rensch (1936), in the first major discussion of Gloger's

333 rule in English, downplayed the role of temperature, placing more importance on humidity

334 (reviewed by Delhey 2019). 
The test of time - and of modern quantitative techniques — have validated Rensch's

336 (1936) emphasis on humidity. Intra- (e.g., Rising et al. 2009, Amar et al. 2014, Marcondes et al.

337 in review) and interspecific (e.g., Delhey 2018, Delhey et al. 2019) comparisons, including this

338 study, have consistently failed to find support for a tendency for birds to be darker in warmer

339 places. Our Models 1 and 2 showed a positive effect of temperature on brightness, particularly in

340 rainy climates (see below). This is diametrically opposite to Gloger's (1833) formulation, but in

341 accordance with intraspecific findings in the Black Sparrowhawk Accipiter melanogaster (Amar

342 et al. 2014), Savannah Sparrow Passerculus sandwichensis (Rising et al. 2009) and Variable

343 Antshrike Thamnophilus caerulescens (Marcondes et al. in review), as well as comparative

344 results from analyses of the Australian avifauna (Delhey 2018) and the world's passerines

345 (Delhey et al. 2019). These findings are consistent with Bogert's rule, a lesser known

346 ecogeographical rule usually considered to apply only to ectothermic animals (Clusella-Trullas et

347 al. 2018, Delhey 2018, 2019). This rule predicts animals should be darker in cooler climates to

348 enhance thermoregulation. The consistency of results showing the same pattern in birds suggests

349 that Bogert's rule may be applicable to endothermic animals as well, although we lack an

350 understanding of its mechanistic underpinnings in that case. Experimental work would be better

351 suited to advance our knowledge in that regard (Delhey 2018).

352 Our models showed a strong interaction between precipitation and temperature (Figures 1

353 and 2). In cooler temperatures, the correlation between greater precipitation and lower brightness

354 was stronger than in warmer temperatures. For illustration, consider four species of furnariids,

355 each occupying a different climatic regime (Figure 2): the Peruvian Recurvebill Syndactyla

356 ucayalae (warm/rainy), the Necklaced Spinetail Synallaxis stictothorax (warm/dry), the Itatiaia

357 Spinetail Asthenes moreirae (cool/rainy), and the Cream-rumped Miner Geositta isabellina

358 (cool/dry). Our results suggest that the two species inhabiting dry climates are expected to be 
brighter than the two species inhabiting rainy climates. But the difference in brightness between the species inhabiting a cool/dry and a cool/rainy climate should be greater than the difference in brightness between the species inhabiting a warm/dry and a warm/rainy climate. This is indeed what our data show. The difference in the first principal component of dorsal brightness between Geositta isabellina (cool/dry) and Asthenes moreirae (cool/rainy) was 0.3328 , whereas the difference in the first principal component of dorsal brightness between Synallaxis stictothorax (warm/dry) and Syndactyla ucayalae (warm/rainy) was 0.1544. support for Gloger's rule for precipitation and Bogert's rule for temperature across the world's passerines, but did not test for their interaction. Delhey et al. (2019) proposed a general framework whereby the effect of temperature on plumage brightness has a quadratic shape, with birds being brighter at low and high temperatures and darker in intermediate temperatures, given

371 the same levels of precipitation. Due to the credible interaction effect we found, our results do

372 not conform to that framework. Instead, they suggest a more nuanced scenario: birds are lighter

373 in cool and dry climates, especially for the ventral plumage, but in cool and rainier climates the

374 effect of precipitation becomes more prevalent, leading to darker plumage (Figure 2). The

375 difference between ours and Delhey et al.'s (2019) conclusions highlights how findings at a more

376 broadly inclusive level (all passerines) may not be directly translatable to a more restricted clade

377 (Furnariidae). This may be because the furnariids include proportionally fewer species occupying

378 very cold climates relative to the passerines as a whole. The minimum temperature in our dataset

379 was $1.7^{\circ} \mathrm{C}$, whereas in Delhey et al.'s dataset it was lower than $-10^{\circ} \mathrm{C}$. Those species from very

380 cold climates, which are also usually dry climates, could be driving the results observed when

381 considering all passerines. 


\section{Gloger's rule, precipitation and habitat type}

Numerous studies have shown that bird species of dark light environments (e.g. forests) tend to be darker than their relatives from open habitats, a pattern attributed to natural selection for crypsis (Endler 1993, McNaught and Owens 2002, Gomez and Thery 2004, Dunn et al. 2015, Maia et al. 2016, Shultz and Burns 2017, Marcondes and Brumfield 2019), but these studies have been conducted largely separately from investigations of Gloger's rule (e.g., Delhey 2018, variables. These contrasts showed that species from bright light environments (nonforest) are credibly brighter, ventrally, than those from intermediate light environments (forest edge and the dorsal plumage, except that there was no difference between nonforest and intermediate 396 habitats (Figure 3). mechanism responsible for Gloger's rule. The aforementioned comparative work and our results

399 corroborate this. Birds tend to be darker in darker (forested) habitats. Because forest habitats also

400 tend to receive more precipitation (for example, precipitation in our dataset, mean $\pm \mathrm{sd}$ : forest

401 species, $2009 \pm 611 \mathrm{~mm} /$ year; intermediate habitat, $1597 \pm 700 \mathrm{~mm} /$ year; nonforest $852 \pm 631$

$402 \mathrm{~mm} / \mathrm{year}$ ), the correlation between brightness and habitat could be spuriously driven by climate.

403 Our results show that is not the case. The difference in brightness across habitats persists even

404 after controlling for climatic variables, demonstrating that they have separate effects on the 405 evolution of plumage brightness. 
Zink and Remsen (1986) also suggested that "humidity per se presumably has little direct

407 influence". Because our Model 2 showed negative correlations between brightness and

408 precipitation, even while including habitat as a predictor, we disagree. Higher precipitation, by

409 itself, does correlate with darker birds. A potential explanation for this is protection against

410 feather-degrading bacteria. It is well-documented that increased melanization makes feathers

411 more resistant to feather-degrading bacteria (Goldstein et al. 2004, Gunderson et al. 2008), and

412 that these bacteria are common on plumages of wild birds (Burtt and Ichida 1999, 2004, Kent

413 and Burtt 2016). However, before it can be conclusively said that feather-degrading bacteria

414 drive increased pigmentation in birds living in rainier habitats, evidence is needed that these

415 bacteria are in fact more abundant in more rainy habitats.

418 Delhey (2018) used remote sensing data to show that, in Australia, birds tend to be darker

419 in more heavily-vegetated areas. This is similar to, and consistent with, our findings. But our

420 analyses based on habitat preference offer further insight, because bird species occupy habitat

421 types differentially even within the same locality, a pattern that cannot be captured by remote

422 sensing-based metrics of vegetation cover. For example, at a typically used resolution, remote

423 sensing data may show that a $30 \mathrm{~m}$ x $30 \mathrm{~m}$ cell is covered in very dense, tall vegetation

424 (rainforest). But different species of furnariids occupying that cell may experience diverse light

425 environments. For example, in western Amazonia that cell may be occupied by the Orange-

426 fronted Plushcrown Metopothrix aurantiaca in the intensely sun-lit forest canopy and the

427 Tawny-throated Leaftosser Sclerurus mexicanus in undergrowth vegetation near the forest floor

428 in the dim forest interior. 

museum specimens collected up to a few decades ago, before recent intense anthropogenic

431 landscape change that will be reflected in remote sensing data. The landscape where a bird was

432 collected many years ago may have little resemblance to the landscape at the same locality today.

433 Nevertheless, vegetation density by itself might also favor increased pigmentation,

434 because greater melanin content makes feathers harder and more resistant to abrasion

435 (Barrowclough and Sibley 1980, Burtt 1986, Bonser 1995). This is often considered in the

436 context of abrasion from airborne particles, but it is conceivable that abrasion from vegetation

437 might also be an important selective factor favoring heavier plumage pigmentation (Kale 1966,

438 Burtt 1986, Surmacki et al. 2011, Kroodsma and Verner 2013), although this demands further 439 empirical study.

\section{Conclusion}

Gloger's rule is a classic ecogeographic principle predicting animals should be darker in

443 wetter and warmer regions. We have shown, based on comparative analyses of the Furnariidae, a

444 family of $>200$ Neotropical passerine species, that the prediction related to precipitation is borne

445 out in our data, but the prediction related to temperature is not. In fact, we found that furnariids

446 tend to be darker in cooler regions. We also found a previously undescribed credible interaction

447 of precipitation and temperature, whereby the negative effect of precipitation on plumage

448 brightness becomes stronger under cool temperatures. Furthermore, we also showed that species

449 in this family tend to be darker in darker light environments and that this effect persists even

450 after controlling for the effects of climate.

$451 \quad$ Based on ours and previous results, we suggest that the pattern encapsulated by Gloger's

452 rule is produced by a combination of the partially correlated effects of habitat type, precipitation, 
and vegetation density. The effect of habitat type is driven by natural selection for enhanced

454

455

456

457

458

459

460

461

462

463

464

465

466

467

468

469

470

471

472

473

474

475

476

crypsis in darker light environments (Zink and Remsen 1986, Endler 1993, McNaught and

Owens 2002, Gomez and Thery 2004, Dunn et al. 2015, Maia et al. 2016, Shultz and Burns

2017, Marcondes and Brumfield 2019), whereas the effect of precipitation may be due to feather-

degrading bacteria (Burtt and Ichida 1999, 2004, Goldstein et al. 2004, Gunderson et al. 2008,

Kent and Burtt 2016), and the effect of vegetation density may be related to feather abrasion

(Kale 1966, Burtt 1986, Surmacki et al. 2011, Kroodsma and Verner 2013), though the latter two

effects still demand further empirical work to be conclusively demonstrated. It is also still

unclear how the effects of temperature fit into this scenario.

\section{References cited}

Amar, A., Koeslag, A., Malan, G., Brown, M. \& Wreford, E. (2014). Clinal variation in the morph ratio of Black Sparrowhawks Accipiter melanoleucus in South Africa and its correlation with environmental variables. Ibis, 156, 627-638.

Barrowclough, G.F. \& Sibley, F.C. (1980). Feather Pigmentation and Abrasion: Test of a Hypothesis. Auk, 97, 881-883.

Bürkner, P.- C. (2017). brms: An R Package for Bayesian Multilevel Models Using Stan. Journal of Statistical Software, 80 .

Burtt, E.H. (1986). An Analysis of Physical, Physiological, and Optical Aspects of Avian

Coloration with Emphasis on Wood-Warblers. Ornithological Monographs, iii-126.

Burtt, E.H. \& Ichida, J.M. (1999). Occurrence of Feather-Degrading Bacilli in the

Plumage of Birds. Auk, 116, 364-372.

Burtt, E.H. \& Ichida, J.M. (2004). Gloger's Rule, Feather-Degrading Bacteria, and Color

Variation Among Song Sparrows. Condor, 106, 681-686. 
Carpenter, B., Gelman, A., Hoffman, M.D., Lee, D., Goodrich, B., Betancourt, M. et al.

478 (2017). Stan: A Probabilistic Programming Language. Journal of Statistical Software, 76.

479 Clusella Trullas, S., van Wyk, J.H. \& Spotila, J.R. (2007). Thermal melanism in

480 ectotherms. J. Therm. Biol., 32, 235-245.

de Villemereuil, P., Wells, J.A., Edwards, R.D. \& Blomberg, S.P. (2012). Bayesian

482

483

484

485

486

487

488

489

490

491

492

493

494

495

496

497

498

499

500 models for comparative analysis integrating phylogenetic uncertainty. BMC Evolutionary

Biology, 12, 1-16.

Delhey, K. (2017). Gloger's rule. Curr. Biol., 27, R689-R691.

Delhey, K. (2018). Darker where cold and wet: Australian birds follow their own version of Gloger's rule. Ecography, 41, 673-683.

Delhey, K. (2019). A review of Gloger's rule, an ecogeographical rule of colour:

definitions, interpretations and evidence. Biol. Rev. Camb. Philos. Soc., 94, 1294-1316.

Delhey, K., Dale, J., Valcu, M. \& Kempenaers, B. (2019). Reconciling ecogeographical rules: rainfall and temperature predict global colour variation in the largest bird radiation. Ecol. Lett., 22, 726-736.

Diniz, P., Ribeiro, P.H.L., Rech, G.S. \& Macedo, R.H. (2016). Monochromatism, cryptic sexual dimorphism and lack of assortative mating in the Rufous Hornero, Furnarius rufus albogularis. Emu, 116, 294-300.

Dunn, P.O., Armenta, J.K. \& Whittingham, L.A. (2015). Natural and sexual selection act on different axes of variation in avian plumage color. Sci Adv, 1, e1400155.

Endler, J.A. (1993). The Color of Light in Forests and Its Implications. Ecol. Monogr., $63,1-27$.

Gelman, A. (2006). Prior distributions for variance parameters in hierarchical models.

Bayesian Analysis, 1, 515-533. 
502 criteria for Bayesian models. Statistics and computing, 24, 997-1016.

503 Goldstein, G., Flory, K.R., Browne, B.A., Majid, S. \& Ichida, J.M. (2004). Bacterial

504 degradation of black and white feathers. Auk, 121, 656-659.

505 Gomez, D. \& Théry, M. (2004). Influence of ambient light on the evolution of colour 506 signals: comparative analysis of a Neotropical rainforest bird community. Ecol. Lett., 7, 279507284.

509 melanized feathers to bacterial degradation: is it really so black and white? J. Avian Biol., 39, $510 \quad 539-545$.

512 resolution interpolated climate surfaces for global land areas. Int. J. Climatol., 25, 1965-1978.

$513 \quad$ James, F.C. (1991). Complementary Descriptive and Experimental Studies of Clinal

514 Variation in Birds. Integr. Comp. Biol., 31, 694-706.

515 Kale, H.W. (1966). Plumages and molts in the Long-billed Marsh Wren. Auk, 83, 140516141.

517 Kamilar, J.M. \& Bradley, B.J. (2011). Interspecific variation in primate coat colour 518 supports Gloger's rule. J. Biogeogr., 38, 2270-2277.

519 Kay, M. (2018). tidybayes: Tidy data and geoms for Bayesian models. R package version 5201.

521 Kent, C.M. \& Burtt, E.H., Jr. (2016). Feather-degrading bacilli in the plumage of wild 522 birds: prevalence and relation to feather wear. Auk, 133, 583-592. 
Kroodsma, D. E. and J. Verner (2013). Marsh Wren (Cistothorus palustris), version 2.0.

524 In: The Birds of North America (A. F. Poole, Editor). Cornell Lab of Ornithology, Ithaca, NY,

525 USA. https://doi.org/10.2173/bna.308

526

Kruschke, J.K. (2013). Bayesian estimation supersedes the $t$ test. Journal of Experimental

527 Psychology: General, 142, 573.

528 Gloger, C.W. (1833). Das Abändern der Vögel durch Einfluss des Klima’s. August

529 Shultz, Breslau.

530 Maia, R., Rubenstein, D.R. \& Shawkey, M.D. (2016). Selection, constraint, and the

531 evolution of coloration in African starlings. Evolution, 70, 1064-1079.

532 Marcondes, R.S. \& Brumfield, R.T. (2019). Fifty shades of brown: Macroevolution of

533 plumage brightness in the Furnariida, a large clade of drab Neotropical passerines. Evolution, 73, $534 \quad 704-719$.

535 Mayr, E. (1956). Geographical Character Gradients and Climatic Adaptation. Evolution, $53610,105-108$.

539 Raton, Florida, USA.

540 McGraw, K.J., Safran, R.J. \& Wakamatsu, K. (2005). How feather colour reflects its

541 melanin content. Funct. Ecol., 19, 816-821.

542 McNaught, M.K. \& Owens, I.P.F. (2002). Interspecific variation in plumage colour

543 among birds: species recognition or light environment? J. Evol. Biol., 15, 505-514.

544 Meiri, S. (2011). Bergmann's Rule--what's in a name? Glob. Ecol. Biogeogr., 20, 203545207. 
Miller, E.T., Leighton, G.M., Freeman, B.G., Lees, A.C. \& Ligon, R.A. (2019).

547 Ecological and geographical overlap drive plumage evolution and mimicry in woodpeckers. Nat.

548 Commun., 10, 1602.

Remsen, J. V. (2003). Family Furnariidae (ovenbirds). Pp. 162-357 in J. del Hoyo, A.

550 Elliott, and D. A. Christie, eds. Handbook of the birds of the world. Vol. 8. Lynx Edicions,

551 Barcelona, Spain.

Rensch, B. (1929). Prinzip geographischer rassenkreise und das problem der artbildung.

553 Gebrüder Borntraeger, Berlin.

Rising, J.D., Jackson, D.A. \& Fokidis, H.B. (2009). Geographic Variation in Plumage

555 Pattern and Coloration of Savannah Sparrows. Wilson J. Ornithol., 121, 253-264.

557 evolution of plumage colour in a cosmopolitan bird. Glob. Ecol. Biogeogr., 28, 496-507.

558 Roulin, A. \& Randin, C. (2015). Gloger's rule in North American barn owls. Auk, 132,

$559 \quad 321-332$.

560

Roycroft, E.J., Nations, J.A. \& Rowe, K.C. (2019). Environment predicts repeated body

561 size shifts in a recent radiation of Australian mammals. Evolution, 74, 671-680.

562 Seeholzer, G.F., Claramunt, S. \& Brumfield, R.T. (2017). Niche evolution and

563 diversification in a Neotropical radiation of birds (Aves: Furnariidae). Evolution, 71, 702-715.

564 Shultz, A.J. \& Burns, K.J. (2017). The role of sexual and natural selection in shaping

565 patterns of sexual dichromatism in the largest family of songbirds (Aves: Thraupidae).

566 Evolution, 71, 1061-1074.

567 Stanchak, K.E. \& Santana, S.E. (2019). Do ecogeographical rules explain morphological

568 variation in a diverse, Holarctic genus of small mammals? J. Biogeogr., 46, 110-122. 
Stoddard, M.C., Sheard, C., Akkaynak, D., Yong, E.H., Mahadevan, L. \& Tobias, J.A.

570 (2019). Evolution of avian egg shape: underlying mechanisms and the importance of taxonomic

571 scale. Ibis, 161, 922-925.

572 Surmacki, A., Liu, M., Mercadante, A. \& Hill, G.E. (2011). Effect of feather abrasion on

573 structural coloration in male eastern bluebirds Sialia sialis. J. Avian Biol., 42, 514-521.

574 Tate, G.J. \& Amar, A. (2017). Morph specific foraging behavior by a polymorphic raptor

575 under variable light conditions. Scientific Reports 7, 9616.

576 Tobias, J.A., Brawn, J.D., Brumfield, R., Derryberry, E.P., Kirschel, A.N.G. \& Seddon,

577 N. (2012). The importance of suboscine birds as study systems in ecology and evolution. Ornitol.

578 Neotrop., 23, 261-274.

579 VanderWerf, E.A. (2012). Ecogeographic patterns of morphological variation in elepaios

580 (Chasiempis spp.): Bergmann's, Allen's, and Gloger's rules in a microcosm. Ornithol. Monogr., $58173,1-34$.

582 Vehtari, A., Gelman, A. \& Gabry, J. (2018). loo: Efficient leave-one-out cross-validation 583 and WAIC for Bayesian models. R package version, 2, 1003.

Zink, R.M. \& Remsen, J.V., Jr. (1986). Evolutionary processes and patterns of

585 geographic variation in birds. In: Current Ornithology (Ed. Johnston, R. F.) 4, 1-69

586

587

588

589

590 


\section{Dorsal}

Scaled Temperature $=-1$

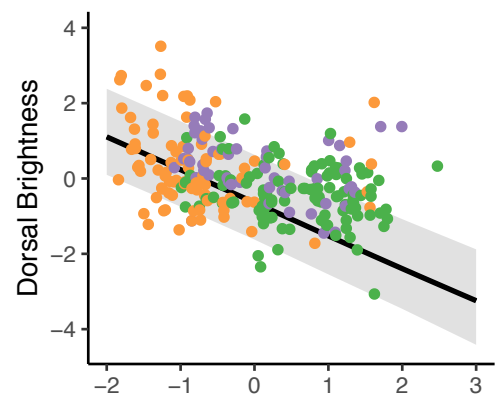

\section{Ventral}

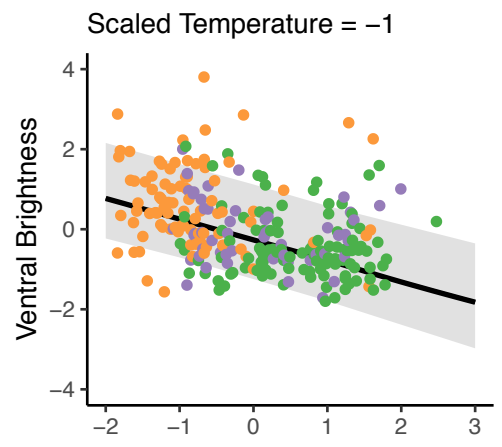

Scaled Temperature $=0$

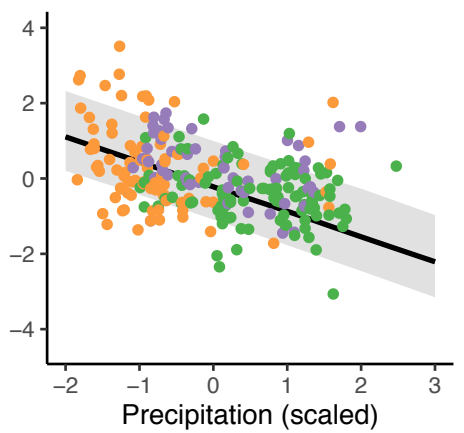

Scaled Temperature $=0$

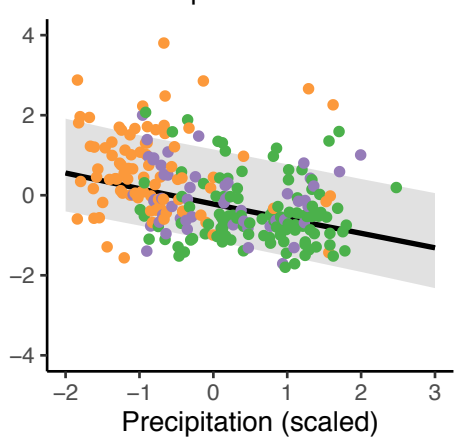

Scaled Temperature $=1$

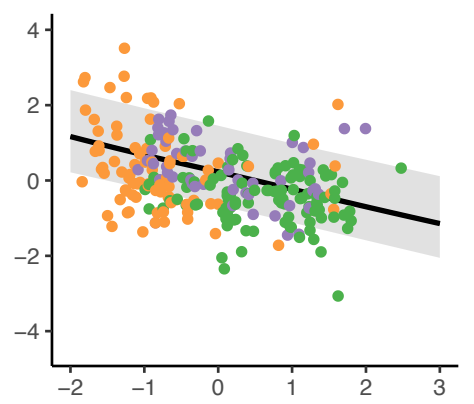

Habitat

Scaled Temperature $=1$

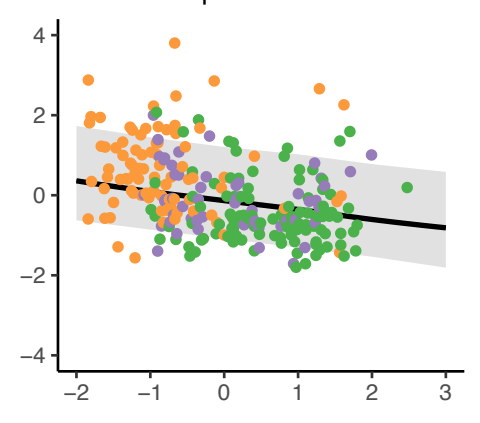

Figure 1. The slope of the negative relationship between temperature and brightness increases as precipitation on brightness when scaled temperature is -1 (mean - 1 standard deviation); the middle column shows the effect of precipitation on brightness when scaled temperature is zero

599 (the mean), and the right-hand column shows the effect of precipitation on brightness when scaled temperature is 1 (mean $+1 \mathrm{SD})$.

601 


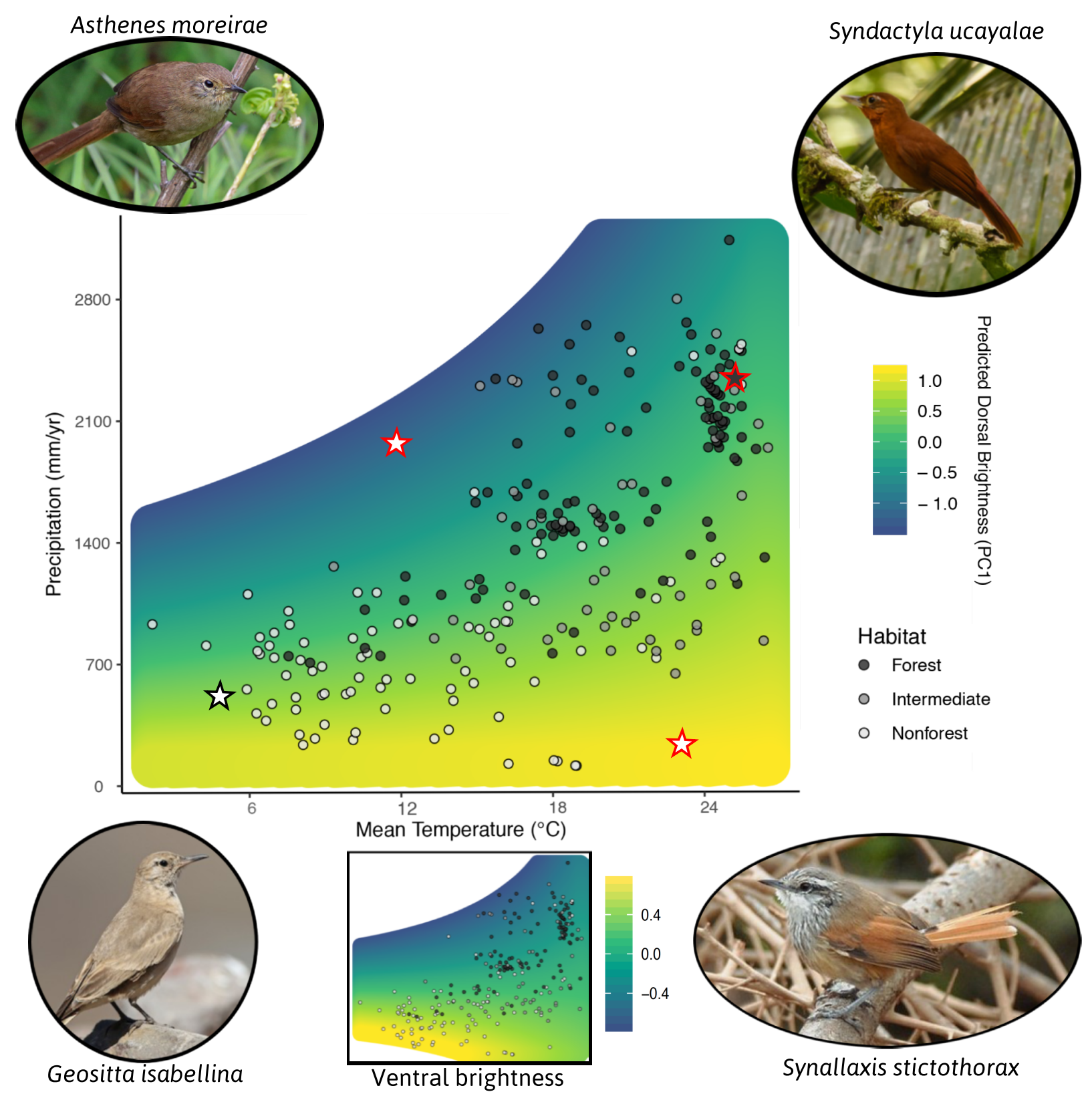

605 Figure 2. A complex interaction between precipitation and temperature predicts dorsal (main

606 panel) and ventral (inset at bottom) brightness in the Furnariidae. The colors in the heatmap

607 represent brightness as predicted by Model 1, which includes precipitation, temperature and their

608 interaction as predictors. Stars represent the depicted species they are closest to. Photograph 
609 authors and licenses: Top left: Nigel Voaden CC BY-SA 2.0. Top right: Rubens Matsushita C.

610 Bottom right: Roger Ahlman (C. Bottom left: Luke Seltz (C.

611

612

613

614

615

616

617

618

619

620

621

622

623

624

625

626

627 


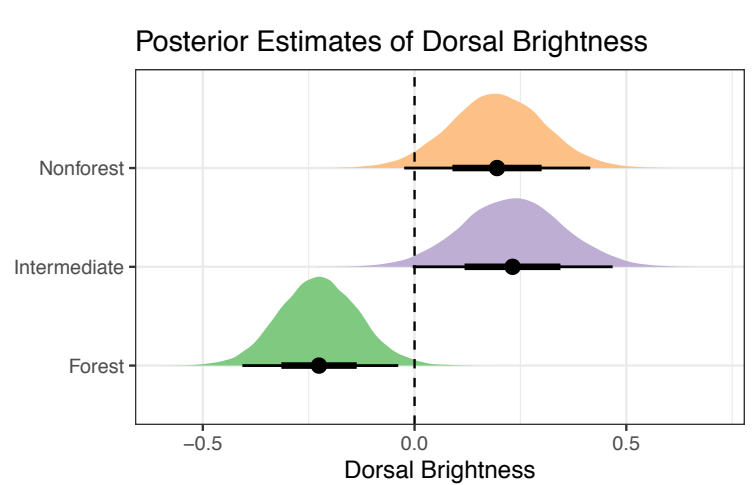

Posterior Estimates of Ventral Brightness
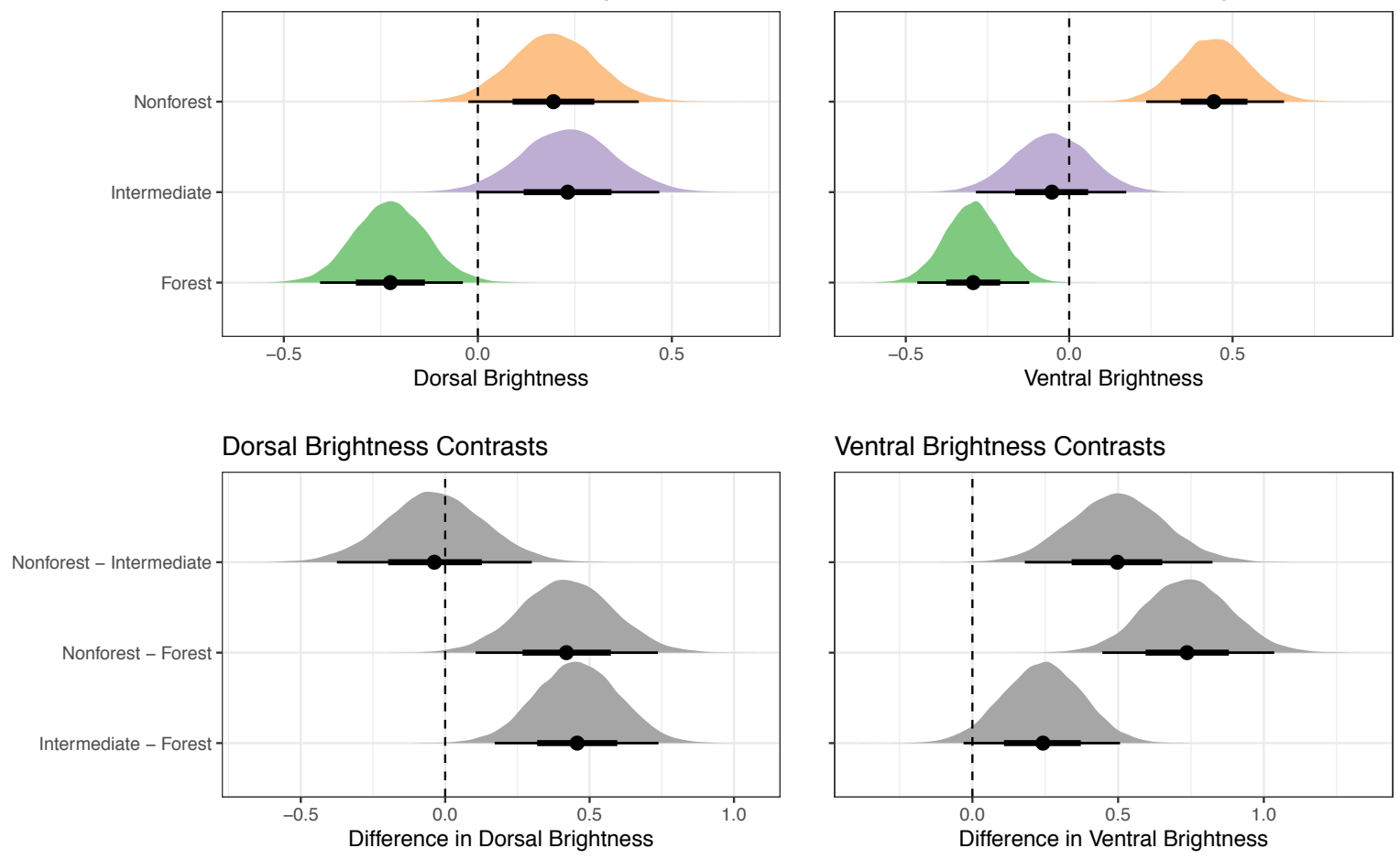

Ventral Brightness Contrasts

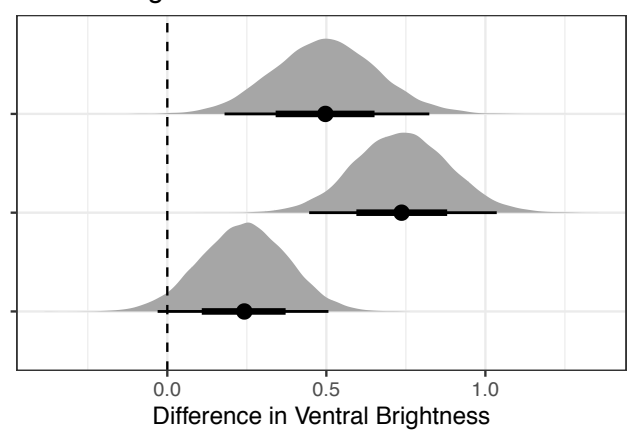

629 Figure 3. Differences in mean plumage brightness across light environments persist even after

630 accounting for climatic variation. Top row: posterior distributions of the mean brightness value,

631 conditioned on temperature, precipitation, and the phylogenetic correlation matrix, for the effects

632 of climate. Bottom row: contrasts between the phylogenetic means of each habitat. If the contrast

633 overlaps zero (dotted line), then there is no credible difference between the brightness of the two

634 habitats. The black circle represents the mean and the horizontal bars the $66 \%$ (thick bar) and

$63595 \%$ (thin bar) credible intervals of the distribution. 


\section{Dorsal Model 1}

\begin{tabular}{lllll}
\hline Population-Level Effects & $\boldsymbol{\alpha}$ mean & $\boldsymbol{\alpha} \mathbf{9 5 \%}$ CI & $\boldsymbol{\beta}$ mean & $\boldsymbol{\beta}$ 95\% CI \\
Intercept & -0.12 & $(-0.26,0.02)$ & - \\
Temperature & - & - & 0.44 & $(0.08,0.28)$ \\
Precipitation & - & - & -0.66 & $(-0.82,-0.51)$ \\
Temperature * Precipitation & & & 0.21 & $(0.07,0.35)$ \\
Family Parameters & Estimate & $\mathbf{9 5 \%}$ CI & & - \\
Sigma & 0.83 & $(0.66,0.95)$ & - & - \\
Alpha & 2.76 & $(1.93,3.74)$ & - & \\
Group-Level Effects & Estimate & $\mathbf{9 5 \%}$ CI &
\end{tabular}

Ventral Model 1

\begin{tabular}{lllll}
\hline Population-Level Effects & $\boldsymbol{\alpha}$ mean & $\boldsymbol{\alpha} \mathbf{9 5 \%}$ CI & $\boldsymbol{\beta}$ mean & $\boldsymbol{\beta}$ 95\% CI \\
Intercept & -0.08 & $(-0.23,0.07)$ & - & - \\
Temperature & - & - & 0.08 & $(-0.08,0.23)$ \\
Precipitation & & & -0.38 & $(-0.53,-0.23)$ \\
Temperature * Precipitation & & & 0.14 & $(0.00,0.29)$ \\
Family Parameters & Estimate & $\mathbf{9 5 \%}$ CI & & \\
Sigma & 0.91 & $(0.81,1.00)$ & - \\
Alpha & 3.21 & $(2.42,4.04)$ & - & - \\
Group-Level Effects & Estimate & $\mathbf{9 5 \%}$ CI & \\
Phylogenetic Error (sd) & 0.14 & $(0.01, .036)$ &
\end{tabular}


643 Table 1: Results from Dorsal Model and Ventral Model 1, where plumage brightness is predicted

644 by temperature, precipitation, and their interaction. $\alpha$ represents the intercept and $\beta$ represents

645 the regression coefficient conditioned on the phylogenetic correlation matrix. Population-Level

646 Effects are the climatic parameters, Sigma is the residual error in the model, Alpha is the skew

647 parameter in the skew-normal distribution, and Phylogenetic Error is the error in the model

648 attributed to the phylogenetic correlation matrix.

649

650

651

652

653

654

655

656

657

658

659

660

661

662

663

664

665 


\section{Dorsal Model 2}

\begin{tabular}{lll}
\hline Population-Level Effects & $\boldsymbol{\beta}$ mean & $\boldsymbol{\beta} \mathbf{9 5 \%}$ CI \\
Precipitation & -0.48 & $(-0.64,-0.32)$ \\
Temperature & 0.35 & $(0.20,0.50)$ \\
Forest Habitat & -0.23 & $(-0.41,-0.04)$ \\
Intermediate Habitat & 0.23 & $(0.00,0.47)$ \\
Nonforest Habitat & 0.20 & $(-0.02, .041)$ \\
Family Parameters & Estimate & $\mathbf{9 5 \%}$ CI \\
Sigma & 0.81 & $(0.65,0.94)$ \\
Alpha & 2.70 & $(1.86,3.69)$ \\
Group-Level Effects & Estimate & $\mathbf{9 5 \%}$ CI \\
Phylogenetic Error (sd) & 0.29 & $(0.02,0.55)$
\end{tabular}

\section{Ventral Model 2}

\begin{tabular}{lll}
\hline Population-Level Effects & $\boldsymbol{\beta}$ mean & $\boldsymbol{\beta} \mathbf{9 5 \%}$ CI \\
Precipitation & -0.19 & $(-0.34,-0.04)$ \\
Temperature & 0.10 & $(-0.05,0.25)$ \\
Forest Habitat & -0.29 & $(-0.46,-0.12)$ \\
Intermediate Habitat & -0.05 & $(-0.29,0.17)$ \\
Nonforest Habitat & 0.44 & $(0.24,0.66)$ \\
Family Parameters & Estimate & $\mathbf{9 5 \%}$ CI \\
Sigma & 0.86 & $(0.76,0.96)$ \\
Alpha & 3.09 & $(2.31,3.93)$
\end{tabular}




$\begin{array}{lll}\text { Group-Level Effects } & \text { Estimate } & \mathbf{9 5 \%} \text { CI } \\ \text { Phylogenetic Error }(\mathrm{sd}) & 0.16 & (0.01, .039)\end{array}$

666

667 Table 2: Results from Dorsal Model and Ventral Model 2, where brightness is predicted by

668 temperature, precipitation and habitat type. $\beta$ represents the regression coefficient, conditioned

669 on the phylogenetic correlation matrix. Population-Level Effects are climatic parameters and the

670 brightness estimates for each habitat. Sigma is the residual error in the model, Alpha is the skew

671 parameter in the skew-normal distribution, and Phylogenetic Error is the error in the model

672 attributed to the phylogenetic correlation matrix.

673

674

675

676

677

678

679

680

681

682

683

684

685

686

687 


\begin{tabular}{|c|c|c|c|}
\hline $\begin{array}{l}\text { Model } \\
\text { Comparison }\end{array}$ & WAIC (se) & ELPD (se) & ELPD Difference \\
\hline $\begin{array}{l}\text { Dorsal Null } \\
\text { Model }\end{array}$ & $693.7(30.8)$ & $-348.8(15.4)$ & $-34.4(9.2)$ \\
\hline Dorsal Model 1* & $629.3(26.2)$ & $-314.6(13.1)$ & $-2.2(4.0)$ \\
\hline Dorsal Model 2* & $624.9(27.5)$ & $-312.4(13.7)$ & $\mathbf{0}$ \\
\hline $\begin{array}{l}\text { Ventral Null } \\
\text { Model }\end{array}$ & $676.8(22.3)$ & $-338.4(11.2)$ & $-23.8(7.6$ \\
\hline Ventral Model 1 & $648.4(24.8)$ & $-324.2(12.4)$ & $-9.5(5.0)$ \\
\hline Ventral Model 2* & $629.3(24.9)$ & $-314.6(12.4)$ & $\mathbf{0}$ \\
\hline
\end{tabular}

Table 3: Model comparison for Dorsal Models and Ventral Models. The first column shows the

691 Widely Applicable Information Criterion (WAIC) score with the standard error of the estimate in

692 parentheses. The second model shows the Estimated Log-Predictive Density (ELPD), or the

693 difference in the model's predictive accuracy, with standard error in parentheses. The third

694 column provides the difference between the ELPD scores and the model with the highest

695 predictive accuracy. In Model 1, brightness is predicted by temperature, precipittaion and their

696 interaction. In Model 2, brightness is predicted by temperature, precipitation and habitat type.

697 For both venter and dorsum, Models 2 (in bold) have the highest predictive accuracy for the

698 given data, and the null models have the lowest predictive accuracy. In the Dorsal models, the

699 standard error of the ELPD difference for Models 1 and 2 overlaps, which means we cannot

700 determine which of the two models has the highest ELPD score. 
bioRxiv preprint doi: https://doi.org/10.1101/2020.04.08.032417; this version posted April 9, 2020. The copyright holder for this preprint (which was not certified by peer review) is the author/funder. All rights reserved. No reuse allowed without permission.

703 